\section{How fast does Halley spin?}

\section{Philip Campbell}

Astonishingly, the rotation rate of the nucleus of comet Halley has suddenly become the focus of intense debate, eight months after the famous spacecraft encounters with the comet. The fact that significant strides have been achieved in the analysis of spacecraft and ground-based photometry and spectroscopy was clear from a recent meeting*. But above all, many participants still seemed to be recovering from the announcement at the previous week's Halley symposium in Heidelberg of the detection of a period in light curves more than three times longer than that hitherto adopted for the nuclear rotation.

The simplest way to investigate cometary rotation, given the acute difficulty in resolving cometary nuclei from Earth, is to seek regular variations in brightness. Because of the vagaries of the allocation of telescope time, there are no light curves available that are continuous since the comet's first detection, making an unbiased Fourier or maximum entropy analysis impractical. One extended collection of data was discussed by M. J. S. Belton (Kitt Peak) but, in reporting a period of 54 hours, he emphasized that he was guided by the selection of possible periods by two previous results - observations from the Japanese spacecraft Suisei of a 2.2-day (53-hour) variability in the ultraviolet intensity of the vast hydrogen cloud surrounding Halley, and an analysis by Z. Sekanina and S. Larson of 1910 dust jet images.

The more sophisticated way to measure the rotation rate of a nucleus is to send three spacecraft to look at it. On 6 March, Vega 1 saw the long axis of the nucleus pointing in a Sunward direction. Three days later, Vega 2 passed Sunward of the nucleus and obtained a broadside image. The two images were consistent with a 480 degree rotation about a spin axis perpendicular both to the long axis of the nucleus and to the orbital plane, as would accord with a 53-hour period. More recently the Giotto and Vega images were considered in combination by both imaging teams who independently arrived at a 53-hour period.

For much of the Heidelberg meeting the cometary scientists had been happy with this rotatory paradigm. But the harmony was broken somewhat when R. L. Millis and co-workers from the Lowell Observatory announced the detection of a strong 7.4-day period in ground-based photometric observations using emission bands

-Annual meeting of the Division of Planetary Sciences of the American Astronomical Society, Paris, 4-7 November 1986. such as $\mathrm{C}_{2}, \mathrm{CN}$ and $\mathrm{OH}$ (Nature, in the press). The light curves, measured in March and April this year, reveal a characteristic double-peaked structure which Millis identified as that produced by active regions entering sunlight once every rotation period.

No-one questions the reality of the 7.4day period in the data - it has now been reported by other observers. For example M. F. A'Hearn et al. (Maryland), following their unexpected discovery of the existence of spiral jets of $\mathrm{CN}$ thought to be the photodissociation product of dust emissions (Nature, in the press), have found that a 7.4-day period and not a 2.2-day period satisfies the relative orientations of the jets at different times. In contrast, $B$. Smith (Arizona) of the Vega imaging team is adamant that the orientations of the nucleus at the three spacecraft encounters cannot accommodate such a period. It is all the more intriguing, therefore, that another member of the Vega team, J. L. Bertaux (Service d'Aeronomie, Verrières-le-Buisson) is less categorical. Nevertheless it does seem that proponents of the 7.4-day rotation period, if they are to conform to the imaging constraints, will have to appeal to a rotation axis more inclined to the long axis of the nucleus and a more complicated nuclear geometry than has hitherto been thought necessary.

As it happens there is still scope for further image enhancement. If the 2.2-day rotation period survives such scrutiny, what can be made of the 7.4-day light curve variations? Their regularity points to some dynamical origin and that most widely assumed seems to be a free wobble (a motion not maintained by a torque) equivalent to the terrestrial Chandler wobble of the Earth, though much closer to the rotation rate of the comet than is the rate of terrestial Chandler wobble to the rotation rate of the Earth. At Heidelberg, K. Wilhelm of the Max Planck Institute, Lindau, who had analysed the motion of an appropriate-sized ellipsoid, reported that a wobble period of about six days was not unreasonable and was consistent with the spacecraft images.

Despite the fact that such a wobble could not be observed directly from outside the cometary coma, its behaviour, if manifested in photometric light curves, could yield useful information about the interior of the nucleus. The wobble would presumably be excited by the jet activity as the comet passed through the vicinity of perihelion. Over the subsequent orbital period, as shown by Wilhelm, it should be damped out by internal friction. But in
Paris, M. Festou (Observatoire de Besançon) presented an amalgamation of all available photometric data taken between January 1984 and February 1985, showing that a 7.4-day period was significant throughout. As J. Lissauer (U. C. Santa Barbara) emphasized, interpreting this as a wobble would imply a significant amplitude near the very onset of emissions from the nuclei, in turn implying small internal damping and a comparatively rigid nuclear interior.

Although such detailed studies of the behaviour of Halley have proved unexpectedly engrossing - and may remain so for some time - more wide-ranging questions were also addressed in Paris, relating in particular to the vast but putative Oort cloud of comets from which Halley is thought to have originated. The darkness of cometary nuclei, emphasized by spacecraft images of Halley, combined with the new findings of the shape and degree of activity of the nucleus, allowed P. Weissman (Jet Propulsion Laboratory) to generalize and estimate afresh the total mass of the cloud, increasing it over a thousandfold above Oort's original estimate of a tenth of an Earth mass or less. This could imply that more comets have collided with Earth than hitherto thought. also strengthening the idea that an early generation of comet-like objects may have delivered the stuff from which the atmospheres and oceans to be found on the terrestrial planets are derived.

The perturbations of the Oort cloud thought to produce long-period comets have traditionally been ascribed to random encounters with passing stars. Recent theoretical work, however, indicates that ever-present galactic tidal forces should also play a significant role. A.H. Delseume and M. Patmiou (Toledo) plotting the aphelia of 152 selected longperiod comets in galactic coordinates, reported 'zones of avoidance' at the Galactic poles and equator, consistent with a tidal effect.

In reviewing the progress in the analysis of spacecraft data, E. Grün (Max Planck Institute, Heidelberg) described surprising results from the PUMA dust impact mass analysers on the Vega spacecraft. Small particles $(<1 \mu \mathrm{m})$ have been found with relative abundances of $\mathrm{C}, \mathrm{N}$ and $\mathrm{O}$ very similar to those in the Sun and much higher than those found in carbonaceous chondritic meteorites. Similarly, gaseous isotope ratios measured from spacecraft and from the ground are consistent with solar abundances. Though the spacecraft results are still preliminary, all the signs are that - as has always been hoped comets contain by far the most primitive and pristine material to be found in the Solar System.

Philip Campbell is Physical Sciences Editor of Nature. 